\title{
L'effet du salaire minimum sur le chômage des jeunes et des femmes au Québec : une réestimation et un réexamen de la question
}

\section{The Effect of the Minimum Wage on Youth and Female Unemployment in Québec: A Reestimation and Reexamination of the Question}

\author{
Jean-Michel Cousineau
}

Volume 67, numéro 2, juin 1991

Symposium sur le salaire minimum et l'emploi

URI : https://id.erudit.org/iderudit/602031ar

DOI : https://doi.org/10.7202/602031ar

Aller au sommaire du numéro

Éditeur(s)

HEC Montréal

ISSN

0001-771X (imprimé)

1710-3991 (numérique)

Découvrir la revue

Citer cet article

Cousineau, J.-M. (1991). L'effet du salaire minimum sur le chômage des jeunes et des femmes au Québec : une réestimation et un réexamen de la question. L'Actualité économique, 67(2), 144-165. https://doi.org/10.7202/602031ar

\section{Résumé de l'article}

Les résultats d'estimation auxquels en arrive cette étude sont à l'effet qu'une hausse du salaire minimum relatif de 10 points de pourcentage contribue à accroître le chômage des jeunes et des femmes au Québec de l'ordre de 1,3 point de pourcentage dans chacun des cas.

La méthodologie utilisée consiste à régresser le taux de chômage de chacun de ces groupes sur des variables d'offre, de demande et de salaire minimum relatif en données semi-annuelles sur la période 1968-1988 pour les femmes et 1976-1988 pour les jeunes.

Par rapport à la littérature existante, on cherche à y préciser la portée des études utilisant le taux de chômage comme variable dépendante, on y re-spécifie les variables d'offre en tenant compte des possibilités de substitution ou de concurrence entre groupe pour les mêmes emplois, on y discute de la prise en compte du chômage structurel, des possibilités de relations non linéaires entre le salaire minimum et le chômage et de la symétrie de ces effets selon qu'on considère une phase de déclin ou de remontée du salaire minimum relatif. Les résultats se montrent très robustes à divers choix de la période d'observation. 


\title{
L'EFFET DU SALAIRE MINIMUM SUR LE CHÔMAGE DES JEUNES ET DES FEMMES AU QUÉBEC: UNE RÉESTIMATION ET UN RÉEXAMEN DE LA QUESTION*
}

\author{
Jean-Michel COUSINEAU \\ École de relations industrielles et \\ Centre de recherche et développement en économique, \\ Université de Montréal.
}

RÉSUMÉ - Les résultats d'estimation auxquels en arrive cette étude sont à l'effet qu'une hausse du salaire minimum relatif de 10 points de pourcentage contribue à accroître le chômage des jeunes et des femmes au Québec de l'ordre de 1,3 point de pourcentage dans chacun des cas.

La méthodologie utilisée consiste à régresser le taux de chômage de chacun de ces groupes sur des variables d'offre, de demande et de salaire minimum relatif en données semiannuelles sur la période 1968-1988 pour les femmes et 1976-1988 pour les jeunes.

Par rapport à la littérature existante, on cherche à y préciser la portée des études utilisant le taux de chômage comme variable dépendante, on y re-spécifie les variables d'offre en tenant compte des possibilités de substitution ou de concurrence entre groupe pour les mêmes emplois, on y discute de la prise en compte du chômage structurel, des possibilités de relations non linéaires entre le salaire minimum et le chômage et de la symétrie de ces effets selon qu'on considère une phase de déclin ou de remontée du salaire minimum relatif. Les résultats se montrent très robustes à divers choix de la période d'observation.

ABSTRACT - The Effect of the Minimum Wage on Youth and Female Unemployment in Québec: A Reestimation and Reexamination of the Question. This article shows that a 10 percentage point change in the relative minimum wages in Québec induces a 1,3 percentage point increase in both the youth and female unemployment rates. The methodology uses the youth (less than 25 years old) and females (all ages) unemployment rates as the dependent variables in a model that considers minimum wages, supply and demand variables as the main explanatory factors. The paper however attempts to re-specifiy the supply variable in order to take account of the competition between youth and females or other groups of labour for the same or similar jobs requiring less or fewer experience in the labour market. It also

* Nous remercions Monsieur Normand Poulet qui nous a grandement aidé à toutes les étapes de la recherche. Une première version de ce texte a aussi grandement bénéficié des commentaires du professeur Claude Felteau de l'Université du Québec à Montréal. Les professeurs François Vaillancourt de l'Université de Montréal et Gilles Grenier de l'Université d'Ottawa nous ont fait plusieurs suggestions utiles. 
discusses the place and significance of such unemployment rate models in the literature, the problems associated with the consideration of structural or «natural» unemployment, the possible non linear type relationship between minimum wages and unemployment, and the question of asymetric effects between periods of high increasing or low decreasing relative minimum wages. The new model shows great robustness and stability over varying sample periods.

\section{INTRODUCTION}

En théorie, l'existence même d'un salaire minimum crée du chômage. En effet, si on suppose que les divers marchés du travail peuvent être caractérisés par une offre et une demande de travail, il arrive alors que la fixation par les autorités gouvernementales d'un salaire minimum au-dessus de l'équilibre de marché augmente les quantités offertes de travail par les travailleurs et réduise les quantités demandées par les employeurs. Il y a donc plus de travailleurs qui offrent leurs services de travail, mais moins d'emplois disponibles sur le marché'.

Sur le plan méthodologique, les économistes ont cherché à vérifier cette hypothèse de deux façons. Certains l'envisagent sous l'angle du chômage, d'autres, sous l'angle de l'emploi. Dans un cas comme dans l'autre, les variables explicatives sont des variables représentant l'offre, la demande et le salaire minimum².

Bien que ces modèles confirment l'existence d'un effet néfaste du salaire minimum sur l'emploi et le chômage ${ }^{3}$, il reste qu'ils comportent un certain nombre de lacunes dont les conséquences peuvent être importantes tant au niveau de la qualité et de la fiabilité des tests qu'à celui des effets estimés.

Un des problèmes importants lié à ces modèles est qu'ils négligent la possibilité de concurrence ou de substitution intergroupe pour les mêmes emplois. C'est ainsi par exemple, que dans l'éventualité où deux groupes seraient en concurrence sur le même marché du travail, la variable d'offre de travail appropriée ne serait pas celle de la population active du groupe visé mais bien celle de l'ensemble des deux groupes. Dans cette étude, nous nous emploierons à vérifier quatre hypothèses de concurrence au niveau de la spécification des variables représentant l'offre de travail dans un modèle de détermination du taux de chômage des jeunes et des femmes au Québec.

1. Pour une revue des théories concurrentes, on peut consulter Grenier et Séguin (1991), West et McKee (1980) et Cousineau (1979).

2. Pour une revue des modèles économétriques utilisés on peut se référer à l'article de Brown $e t$ al. (1982) et, pour une revue des résultats d'estimation on peut se référer à ce même article pour les estimations américaines ainsi qu’à West et McKee (1980) et Gunderson et Riddell (1988) pour les résultats canadiens.

3. Jusqu'à tout récemment, on s'accordait pour dire, par exemple, qu'un accroissement de $10 \%$ du salaire minimum conduisait à une réduction de l'emploi chez les adolescents de l'ordre de $1 \%$ à $3 \%$ (Brown et al. 1982 pour les États-Unis et Gunderson et Riddell 1988 pour le Canda). 
La première section précise la portée et les limites d'une étude ayant le taux de chômage comme variable dépendante. Elle s'appuie et complète essentiellement l'analyse critique qu'ont fait Brown et al. (1982) de ce genre de modèle par rapport aux modèles d'emploi. La seconde et la troisième section procèdent à la respécification puis à l'estimation, dans le modèle, des variables représentant l'offre de travail. On y considère également les problèmes liés à la prise en compte du chômage structurel, à la symétrie des effets du salaire minimum à la hausse et à la baisse, et aux possibilités de relations non linéaires entre le salaire minimum et le chômage. Finalement, et compte tenu de l'importance qu'il y a à avoir des résultats robustes dans ce genre d'estimation, la dernière section procède à une analyse de sensibilité des résultats quant au choix de la période d'observation.

\section{REMARQUES GÉNÉRALES}

Selon Brown et al. (1982, p. 497)4, les études sur le chômage ne mesureraient pas aussi bien le tort fait à l'économie que les études sur l'emploi. La hausse des quantités offertes, présumée par le modèle de base, entraîne du chômage pour ceux et celles qui n'auraient pas offert leurs services de travail n'eut été de la hausse du salaire minimum relatif. Ce type de chômage ne serait pas aussi grave que celui occasionné par des pertes d'emploi. Sur le plan empirique toutefois, il arrive que cette hypothèse n'est pas confirmée. En effet, les coefficients obtenus sur la variable de salaire minimum dans les équations de taux d'activité sont très souvent négatifs (voir, entre autres, Benhayoun 1990). Par ailleurs, le fait que plusieurs travailleurs mis-à-pied se retirent éventuellement du marché du travail contribuerait à sousestimer les effets sur l'emploi.

Cette seconde hypothèse a beaucoup plus de poids car la relation inverse entre les taux d'activité et le taux de chômage est largement confirmée sur le plan empirique (Grenier et Séguin 1991). Dès lors, si le salaire minimum réduit l'emploi mais qu'une partie des travailleurs mis-à-pied ne figure pas parmi les chômeurs, nous devons en conclure que l'utilisation du taux de chômage comme variable dépendante constitue une sous-estimation de ses effets sur le marché du travail.

Deuxièmement, selon Brown et al. (1982, p. 497) toujours,le concept et la statistique du chômage seraient flous et imprécis. En effet, la statistique du chômage ne mesure pas tout le degré de sous-emploi dans une économie. Ceux et celles qui ne cherchent pas activement de l'emploi sur le marché du travail après un certain temps ne sont pas considérés comme chômeurs. D'une part cependant, il faudrait considérer que l'emploi n'est pas moins flou ou imprécis en mettant sur le même pied des emplois temporaires ou à temps partiel avec des emplois réguliers à temps plein. En effet, il suffit de rapporter quelques heures de travail pour être considéré à l'emploi au même titre qu'un emploi à temps plein'.

4. Tous nos commentaires s'appuieront sur l'analyse critique qu'ont fait Brown et al. (1982) relativement aux modèles utilisant le taux de chômage comme variable dépendante.

5. Statistique Canada considère qu'un emploi est à temps partiel si le nombre d'heures travaillées par semaine est de moins de 30 heures. Cela offre donc la possibilité d'étudier séparément l'incidence du salaire minimum sur l'emploi à plein temps et à temps partiel de façon distincte. Dans les études sur l'emploi, très peu font cette distinction toutefois. 
D'autre part, il convient de mentionner que la statistique du chômage essaie de mesurer, autant que possible, le chômage visible, c'est-à-dire de se rapprocher du concept d'offre de travail qui correspond au nombre de personnes qui visiblement offrent leurs services de travail sur les marchés du travail et qui conditionne la dynamique des salaires et l'allocation des ressources humaines sur ces différents marchés. En ce sens, il s'agit d'une statistique relativement adéquate fondée sur des concepts relativement précis. Elle fait d'ailleurs l'objet d'une attention toute particulière de la part des chercheurs, des organismes de décisions privés ou publics, des politiciens et de l'opinion publique en général. Le fait que la statistique officielle du chômage ne tienne pas compte des personnes qui ont décidé de se retirer du marché du travail nous rappelle toutefois que l'étude de l'incidence du salaire minimum sur le chômage constitue une évaluation minimale de ses effets sur l'emploi et le degré de sous-emploi dans une économie.

Troisièmement, les changements d'enquête qui ont eu lieu aux États-Unis en 1967 auraient davantage affecté les statistiques sur le chômage que sur l'emploi (Brown et al, p. 498). Au Canada, l'enquête sur la population active a aussi changé en 1975. Grenier et Séguin (1990) ont convenu toutefois qu'il fallait scinder les données sur le chômage et l'emploi en fonction de périodes d'échantillonnages comparables. Pour notre part, nous avons convenu de nous adresser aux meilleures données comparables, soit la pleine période d'observation (1968-1988) pour les femmes et la sous-période 1976-1988 pour les jeunes ${ }^{6}$.

Quatrièmement, les prédictions théoriques relatives à l'effet du salaire minimum sur le chômage seraient en fait indéterminées pour deux raisons. D'une part, le salaire minimum augmente le chômage qui diminue la population active qui diminue le chômage. L'effet net est donc indéterminé a priori. D'autre part, l'augmentation du salaire minimum réduit l'emploi des plus jeunes et des travailleurs moins qualifiés au profit de travailleurs plus âgés et/ou plus qualifiés, en même temps qu'il diminue l'emploi et la production de façon générale. L'effet sur l'emploi et le chômage des travailleurs adultes est donc lui aussi indéterminé a priori. Nos commentaires sont à l'effet que l'étude empirique ou économétrique de l'incidence du salaire minimum sur le chômage constitue une évaluation de cette incidence, une fois tous ces ajustements complétés?

Cinquièmement et pour en revenir en quelque sorte au premier point, il convient de mentionner que l'étude de l'incidence du salaire minimum sur l'emploi est préférable à celle de son incidence sur le chômage pour autant que la méthodologie utilisée dans les études surl'emploi soit meilleure. Or, il n'est pas évident que ce soit lecas. Telle que présentée par Brown et al.(1982,p. 497), cette méthodologie consiste à régresser le ratio emploi/population sur une variable de demande (cycle des

6. En 1975, l'Enquête sur la population active était révisée. Statistique Canada a été en mesure de fournir des données révisées rétroactivement pour les femmes mais non pour les jeunes.

7. Pour autant que la périodicité des données soit assez longue et que la période d'ajustement soit suffisamment courte. 
affaires), une variable de salaire minimum et un ensemble de variables de contrôle pour l'offre de travail. Le cadre de référence théorique est donc celui du marché, beaucoup plus approprié pour l'étude du chômage, alors qu'il serait nettement plus avantageux et indiqué de se référer à la théorie de la demande pour les facteurs de production.Sur le plan empirique par ailleurs, les travaux sur la détermination de l'emploi qui s'appuient sur ce type de théorie sont fort nombreux et avancés.

En effet, depuis 1965, Brechling, à la suite de Oi(1962), a tout d'abord rendu opérationnelle la notion de main-d'oeuvre en tant que facteur de production quasifixe sous forme de modèle d'ajustement partiel. Dans ce modèle, l'emploi suit donc l'évolution de la production (et non celle du taux de chômage des hommes adultes), d'un terme de tendance reflétant l'évolution de la technologie et de l'emploi retardé d'une période. Le coefficient de cette dernière variable permettant de calculer le taux d'ajustement partiel, il y a donc des avantages à ce que la spécification de l'emploi incorpore la variable endogène retardée au niveau de ses variables explicatives.

En 1968, Nadiri prenait en considération de façon explicite dans ce type de modèle, une variable de salaire et de prix du capital. Et, finalement, Clark et Freeman (1980), à la suite de nombreux travaux sur la question, confirmaient, dans le cadre d'un modèle double-logarithmique de type Cobb-Douglas, l'hypothèse à l'effet que l'ajustement de l'emploi à ces variables suivait un processus d'anticipations (les décisions sur l'emploi se font sur les valeurs anticipées de ces prix) caractérisé par des structures de retards propres à chacune de ces variables.

Par ailleurs, en alternative aux fonctions d'emploi, on a assisté au développement du concept de fonctions logarithmiques transcendentales (Berndt et Christansen 1973) avec pour applications plus récentes la considération explicite des éventuelles possibilités de substitution et de complémentarité entre les différents sous-groupes demain-d'oeuvre parâge, sexe et race (Hamermesh 1988). Toutes ces méthodologies sont disponibles pour l'étude de l'incidence du salaire minimum sur l'emploi et s'avéreraient une approche plus fondée tant sur les plans empiriques que théoriques ${ }^{8}$. À ce titre, on ne peut donc supposer que les études existantes de l'incidence du salaire minimum sur l'emploi sont meilleures que les études existantes relatives à l'incidence du salaire minimum sur le chômage. On peut envisager cependant qu'elles deviennent complémentaires l'une de l'autre et qu'elles jouent un rôle de double et de contre-vérification.

\section{UNE RESPÉCIFICATION DU MODÈLE}

Le fait que l'approche par le chômage se justifie ne signifie pas nécessairement que les études sur le chômage sont exemptes de limites et de lacunes. Dans cette section, nous signalerons quelques-unes de ces lacunes puis nous spécifierons un cadre empirique en mesure d'apporter des améliorations aux modèles existants.

8. Une autre limite à ces études est le fait qu'en données mensuelles ou trimestrielles par province, la qualité des données peut être sérieusement mise en doute. L'erreur d'échantillonnage étant d'autant plus élevée que le groupe de référence (e.g. jeunes de 15 à 19 ans) est petit. 


\section{Lacunes}

Premièrement, il convient de souligner que le modèle de base (offre, demande et salaire minimum) ignore la présence ou l'existence de chômage structurel. En effet, dans ce genre de modèle théorique, tout le chômage est essentiellement attribuable au salaire minimum. Or, dans la mesure où il existe du chômage pour les groupes qui gagnent habituellement plus que le salaire minimum, il est clair qu'il existe d'autres formes de chômage.

Si le chômage structurel ou naturel était constant dans le temps ou encore s'il variait selon une tendance régulière, linéaire ou curvilinéaire, la prise en compte de ce phénomène pourrait se faire par un terme de tendance et un terme constant. Néanmoins, sur la base des travaux sur les taux de chômage structurels ou naturels (cf. Coe 1990 ou Fortin 1989), il n'est pas évident que l'une ou l'autre de ces hypothèses ne conviennent à la prise en compte de ce phénomène. Les composantes structurelles du chômage (assurance-chômage, variabilité interindustrielle de l'emploi, etc.) suivent des mouvements irréguliers dans le temps et il n'est pas établi que leur combinaison adopte un comportement tendanciel régulier. Pour les fins d'estimation, il convient donc de tenir compte d'une certaine façon de l'existence de chômage structurel et de sa variabilité propre dans le temps ${ }^{9}$.

Deuxièmement, et nous insistons particulièrement sur ce point, il importe de noter que l'offre de travail dont il est question dans la détermination du taux de chômage des jeunes ou des femmes n'est pas nécessairement et exclusivement caractérisée par la population active de l'un ou l'autre groupe. Dans un premier temps, il est vrai qu'il apparaît approprié de retenir cette mesure puisque, par identité, le chômage est égal à l'écart entre la population active et l'emploi de ce groupe. Néanmoins, il se peut qu'un groupe soit en concurrence avec un autre pour les mêmes emplois et que ce soit la population active totale de ces deux groupes qui serve de meilleure approximation de l'offre effective de travail sur le marché visé ${ }^{10}$.

La troisième remarque tient au fait que l'effet estimé est égal au produit de l'élasticité de la demande sur le marché du travail des personnes au salaire minimum par le poids qu'occupent ces personnes dans la population active du groupe à l'étude (Mercier 1985). Or, dans la mesure où le salaire minimum relatif influence lui-

9. Théoriquement, ce phénomène peut être pris en compte, en partie tout au moins, en faisant le tracé d'une courbe d'emploi effectif sous la courbe de demande de travail. Les écarts entre la demande de travail et l'emploi effectif traduisent alors les déséquilibres structurels sur le marché du travail.

10. Brown et al. (1982) rappellent qu'au début des années 70 il y eut un débat quant à savoir si les modèles de détermination du chômage devaient ou non tenir compte d'une variable d'offre de travail. Pour les uns on n'avait pas à tenir compte de l'offre parce que son augmentation n'a d'incidence sur le chômage qu'a la condition qu'il existe un salaire minimum rigide et fixe. Autrement, l'offre fait pression à la baisse sur les salaires et il $n$ 'y a pas de chômage additionnel. Néanmoins, comme le font remarquer Brown et al., (p. 501-2), l'étude de l'incidence du salaire minimum sur le chômage se fait pour un niveau d'offre donné. Il devient donc nécessaire d'en tenir compte dans les travaux d'estimation. 
même ce poids, il en ressort qu'une forme non linéaire est plus indiquée qu'une forme linéaire simple ${ }^{11}$. En effet, si par exemple:

$$
T C_{i}=a_{\mathrm{o}}+a_{1} S M R+\sum_{j} a_{j} X_{j}
$$

et que:

$$
a_{1}=b_{\mathrm{o}} S M R,
$$

il s'ensuit, par substitution de (2) dans (1), que:

$$
T C_{i}=a_{\mathrm{o}}+b_{\mathrm{o}} S M R^{2}+\sum_{j} a_{j} X_{j}
$$

où $S M R=$ taux de salaire minimum relatif, $X_{j}=$ autres variables de détermination du chômage et $T C_{i}=$ taux de chômage du groupe $i$. À noter, toutefois, que la forme quadratique simple ( $\left.S M R^{2}\right)$ est arbitraire et qu'il pourrait s'agir d'autres formes non linéaires. Par exemple, on aurait pu choisir $a_{1}=b_{0}+b_{1} S M R$, ce qui aurait généré une forme quadratique différente. Dans les sous-sections qui suivent, nous traduirons ces remarques en termes plus opérationnels mais auparavant, il convient de fournir les grandes données du cadre de vérification empirique.

\section{Les variables dépendantes et l'estimateur choisi}

L'estimateur choisi est les moindres carrés ordinaires appliqués à deux équations de chômage: une pour le taux de chômage des jeunes et une pour le taux de chômage des femmes. La raison de ce choix est que les jeunes de moins de 25 ans et les femmes de tous âges forment la très vaste majorité des personnes au salaire minimum ${ }^{12}$.

\section{La nature des données}

En ce qui a trait au choix des données, nous avons opté pour des données brutes non désaisonnalisées au niveau des variables dépendantes et désaisonnalisées au niveau des variables indépendantes. La saisonnalité dans les variables indépendantes n'étant pas forcément celle des variables dépendantes, nous avons donc préféré cette approche. Une ou des variables dichotomiques saisonnières sont ajoutées au modèle de façon à capter la saisonnalité dans les variables dépendantes.

\section{La périodicité des données}

Afin d'éclairer notre choix relatif à la périodicité des données, nous avons dressé la liste de la périodicité à laquelle était révisé le salaire minimum. Cette liste apparaît au tableau 1.

11. Dans les conditions actuelles, on peut facilement imaginer qu'un salaire minimum de $10 \$$ l'heure couvrirait une plus large proportion de la main-d'ouvre des jeunes et des femmes qu'un salaire de 5,30\$ l'heure.

12. En $1974,68,4 \%$ des personnes au salaire minimum étaient de sexe féminin et $51,8 \%$ avaient moins de 25 ans (Boutin 1974). En 1984, 63,2\% des personnes au salaire minimum étaient de sexe féminin et $\mathbf{2 5 , 7 \%}$ avaient moins de 20 ans (Cournoyer 1988). Nous n'avons pas la statistique pour les moins de 25 ans pour cette année. 
TABLEAU 1

PÉRIODICITÉ DES RÉVISIONS APPORTÉES AU SALAIRE MINIMUM, QUÉBEC, 1968-1988

\begin{tabular}{ll|ll|ll}
\hline \hline Année & $\begin{array}{c}\text { nombre de } \\
\text { révisions }\end{array}$ & Année & $\begin{array}{c}\text { nombre de } \\
\text { révisions }\end{array}$ & Année & $\begin{array}{c}\text { nombre de } \\
\text { révisions }\end{array}$ \\
\hline 1968 & 1 (nov.) & 1975 & 2 (juin, déc.) & 1982 & 0 \\
1969 & 1 (mai) & 1976 & 1 (juillet) & 1983 & 0 \\
1970 & 2 (juin, nov.) & 1977 & 2 (janv., juil.) & 1984 & 0 \\
1971 & 1 (nov.) & 1978 & 2 (janv., oct.) & 1985 & 0 \\
1972 & 2 (août, nov.) & 1979 & 1 (avril) & 1986 & 1 (oct.) \\
1973 & 2 (mai, nov.) & 1980 & 1 (avril) & 1987 & 1 (oct.) \\
1974 & 2 (mai, nov.) & 1981 & 2 (avril, oct.) & 1988 & 1 (oct.) \\
\hline
\end{tabular}

Nombre de révisions semi-annuelles: 8 ;

Nombre de révisions annuelles: 9;

Aucune révision: 4 .

SOURCE: Gazette officielle du Québec

On y observe que dans 4 cas (1982-1985) sur 21, il n'y eut aucune révision du salaire minimum. Par contre, dans à peu près la moitié des cas où le salaire minimum a été révisé, cette révision s'est effectuée soit une fois, soit deux fois par année. Il semble donc que, de façon générale, les politiques de salaire minimum fassent l'objet de considérations en moyenne une ou deux fois par année. Pour les fins de l'étude de l'incidence du salaire minimum sur le chômage, nous en concluons que l'usage de données annuelles ou semi-annuelles apparaît la plus appropriée. Par contre, dans la mesure où des données semi-annuelles offrent plus de degrés de liberté et donc une meilleure précision au niveau des différents tests statistiques, nos estimations s'effectueront sur la base de données semi-annuelles ${ }^{13}$. La variable dichotomique saisonnière $D S$ est donc égale à l'unité pour la deuxième moitié de l'année et à zéro autrement. Elle contrôle pour l'éventualité d'effets saisonniers asymétriques.

\section{Les variables indépendantes}

Le problème de la prise en compte des composantes structurelles et conjoncturelles du chômage des jeunes et des femmes a été abordé de deux façons soit a) en considérant une variable de déviation du PIB québécois (en dollars constants)

13. D'autres raisons appuient ce choix soit 1) la réduction des problèmes d'autocorrélation (l'échantillon de l'Enquête sur la population active est révisé au rythme d'un sixième par mois) et 2) la réduction d'autres sources de bruit tels la saisonnalité du salaire horaire dans le secteur manufacturier (non apparente dans le cas de données semi-annuelles) et surtout un comportement «artificiel en dents de scie» du salaire minimum relatif lorsque des données mensuelles ou trimestrielles sont utilisées. 
exprimée en pourcentage de sa tendance exponentielle à long terme (1961-1988) accompagnée de diverses variables de tendance $\left(t ; t\right.$ et $t^{2}$; ou $t, t^{2}$ et $\left.t^{3}\right)$, ou b) en considérant le taux de chômage total pour l'ensemble de l'économie québécoise $(T C T)$. En effet, dans ce dernier cas, on peut considérer que le taux de chômage des jeunes ou des femmes évolue en fonction des fluctuations du taux de chômage total ainsi qu'en fonction de l'évolution de sa composante structurelle. Le taux de chômage total a le défaut de créer un biais de simultanéité par le fait qu'il partage, avec l'une et l'autre des variables dépendantes, un terme d'erreur d'échantillonnage. Néanmoins, cette dernière variable a été préférée à la première formulation parce qu'elle tient compte des composantes conjoncturelles et structurelles du chômage et qu'elle évite d'avoir à considérer des termes de tendance plus ou moins arbitraires qui captent toutes sortes d'influences, qui entrent en corrélation avec d'autres variables explicatives du modèle et qui, somme toute, gênent l'interprétation des résultats ${ }^{14}$.

Notre définition opérationnelle du salaire minimum relatif est à l'instar des autres travaux sur la question, composée du quotient du salaire minimum sur le salaire horaire moyen des ouvriers de production dans l'industrie manufacturière. Compte tenu du changement d'enquête survenu en mars 1983 au niveau du salaire manufacturier, la première série de salaires sur le secteur manufacturier a été prolongée sur la base des variations de salaires rapportées dans la nouvelle enquête pour la période suivant cette révision. En régressant cette variable sur un terme constant et une variable dichotomique semi-annuelle, nous avons pu vérifier également qu'elle n'avait pas de comportement saisonnier particulier (en données semi-annuelles). La variable de salaire minimum relatif $(S M R)$ a été multipliée par 10 de façon à ce que l'estimation obtenue traduise directement l'effet, sur le taux de chômage, d'une variation de 10 points de pourcentage dans le salaire minimum relatif.

En ce qui a trait à l'hypothèse de non linéarité, diverses formes ont été mises à l'essai: $\log -\log$, semi-log à gauche, semi-log à droite, quadratiques simples $\left(S M R^{2}\right)$ ou complexes $\left(S M R\right.$ et $\left.S M R^{2}\right)$. Les techniques de Box et Cox ont également été mises à contribution.

Finalement, et c'est peut-être le point le plus délicat: puisque la théorie ne nous spécifie pas à l'avance avec quel groupe les jeunes ou les femmes sont en concurrence sur le marché du travail, nous avons laissé le soin aux tests et estimations économétriques de déterminer la variable d'offre la plus pertinente. Quatre hypothèses non imbriquées ont été construites à cet effet, chacune de ces hypothèses ayant sa contrepartie empirique sous forme de spécification propre. Ces quatre hypothèses sont les suivantes: 1- le comportement de l'offre d'un groupe a été parfaitement compensé par celui des autres groupes concurrents; 2 - la concurrence se limite au seul groupe visé; 3-la concurrence s'étend au second groupe à l'étude; 4- la concurrence s'étend à l'ensemble de la main-d'œuvre.

14. Le taux de chômage des hommes adultes a été rejeté a priori sur la base du fait qu'il est trop peu général (ignore une grande partie de l'économie) et comporte les mêmes défauts que l'usage du PIB du fait qu'il nécessite aussi l'introduction de termes de tendance linéaires ou curvilinéaires. 
En termes opérationnels, ces hypothèses se traduisent par $\mathrm{H} 1$ : la variable de population active relative est constante et assimilée au terme constant $\left(P A R_{1}=1\right)$; $\mathrm{H} 2$ : la variable de population active relative est constituée de la seule population active du groupe visé $\left(P A R_{21}=\right.$ population active des jeunes exprimée en pourcentage de la population active totale $=P A R J$ pour les jeunes ${ }^{15}$, et $P A R_{22}=$ population active des femmes exprimée en pourcentage de la population active totale $=P A R F$ pour les femmes); H3: la variable de la population active relative est constituée de la somme des populations actives des jeunes et des femmes (PAJ et PAF respectivement $)$ en pourcentage de la population active totale $\left(P A R_{3}=((P A J+P A F) /\right.$ $\left.P A T)^{*} 100\right) ; \mathrm{H} 4$ : la variable de population active relative est assimilable au taux d'activité global pour l'ensemble du Québec $\left(P A R_{4}=T A\right)$.

A priori, nous pouvons exprimer quelques préférences pour l'une ou pour l'autre de ces hypothèses. En premier lieu, le cadre théorique nous amène à préférer l'hypothèse de concurrence de la main-d'œuvre entre les jeunes et les femmes pour les nouveaux emplois exigeant peu d'expérience(H3). En second lieu par contre, nous pouvons considérer l'hypothèse $\mathrm{H} 2$ à l'effet qu'il existe une certaine segmentation des marchés par sexe et que les jeunes se voient refuser l'accès à certains marchés faute d'expérience. En troisième lieu, nous considérons l'hypothèse d'une concurrence très large et diffuse sur l'ensemble des marchés $(\mathrm{H} 4)$ et, en dernier lieu, nous considérons l' hypothèse de la parfaite compensation des offres de travail $(\mathrm{H} 1)^{16}$.

\section{LES RÉSULTATS D'ESTIMATION}

Le tableau 2 présente l'ensemble des résultats d'estimation suivant cet ordre de préférences. Les statistiques $t$ y apparaissent entre parenthèses sous les coefficients. Les statistiques $\mathrm{R}^{2}$, F et Durbin-Watson (D.-W.) ainsi que le coefficient de corrélation partielle ( $\mathrm{r}$ ) entre la variable de salaire minimum relatif et la variable de population active considérée apparaissent dans les dernières colonnes du tableau. La portion supérieure du tableau rapporte les résultats d'estimation pour les femmes. La portion inférieure rapporte les résultats pour les jeunes et la note en bas de page indique les sources de données. Les données sont semi-annuelles et, sauf

15. Dans le cas de la population active des jeunes, il a fallu procéder par moyenne mobile pour arriver à désaisonnaliser cette série statistique. Statistique Canada ne foumissant pas directement de données désaisonnalisées pour ce groupe de main-d'œuvre. Cela explique également pourquoi nous perdons une année d'observation sur la période 1975-1988 et que nous devons commencer la série à partir de 1976.

16. L'usage de ratios de population en âge de travailler présente des avantages économétriques sur celui des ratios de population active eu égard notamment aux problèmes de simultanéité avec la variable dépendante de même qu'avec la variable de salaire minimum. Néanmoins, la théorie prédisant que le chômage n'est pas affecté par la population active potentielle mais bien par la population active réelle, nous avons préféré nous en tenir à la seconde formulation. Par ailleurs, le lien entre l'offre de maind'cuvre potentielle et l'offre de travail effective est complexe. Dans le cas des femmes, leur taux d'activité a sensiblement augmenté en combinaison avec l'augmentation de leur population en âge de travailler. Dans le cas des jeunes, leurs effectifs démographiques ont diminué en termes absolus alors que leur taux d'activité a eu tendance à s'accrôitre. 
TABLEAU 2

ÉTUDE DE CERTAINES HYPOTHÈSES RELATIVES À LA VARIABLE D'OFFRE DE TRAVAIL ${ }^{17}$

\begin{tabular}{|c|c|c|c|c|c|c|c|c|c|c|}
\hline & $a_{0}$ & $T C T$ & $S M R$ & $P A R_{\downarrow}$ & DS & $\mathrm{R}^{2}$ & $\overline{\mathbf{R}}^{2}$ & F & D.-W. & r \\
\hline \multicolumn{11}{|l|}{ FEMMES } \\
\hline $\begin{array}{l}\text { H3 (Concur- } \\
\text { rencées par } \\
\text { les jeunes)* }\end{array}$ & $\begin{array}{c}-6,85 \\
(-1,44)\end{array}$ & $\begin{array}{c}0,86 \\
(20,79)\end{array}$ & $\begin{array}{c}0,28 \\
(2,59)\end{array}$ & $\begin{array}{c}0,12 \\
(1,55)\end{array}$ & $\begin{array}{c}-0,03 \\
(-0,22)\end{array}$ & 963 & ,955 & 134,9 & 1,95 &, 47 \\
\hline $\begin{array}{l}\text { H2 (ségré- } \\
\text { gation) }\end{array}$ & $\begin{array}{c}-15,36 \\
(-12,22)\end{array}$ & $\begin{array}{c}0,96 \\
(28,11)\end{array}$ & $\begin{array}{c}1,25 \\
(11,37)\end{array}$ & $\begin{array}{c}0,28 \\
(10,07)\end{array}$ & $\begin{array}{c}-0,10 \\
(-1,04)\end{array}$ & ,988 & ,987 & 789,6 & 1,93 &,- 75 \\
\hline $\begin{array}{l}\mathrm{H} 4 \text { (concur- } \\
\text { rence large) }\end{array}$ & $\begin{array}{c}-22,38 \\
(-10,38)\end{array}$ & $\begin{array}{c}1,05 \\
(33,34)\end{array}$ & $\begin{array}{c}1,09 \\
(9,73)\end{array}$ & $\begin{array}{c}0,29 \\
(8,86)\end{array}$ & $\begin{array}{c}-0,12 \\
(-1,14)\end{array}$ & ,986 & ,985 & 657,3 & 1,85 &,- 70 \\
\hline $\begin{array}{l}\text { H1 (compen- } \\
\text { sation) }\end{array}$ & $\begin{array}{c}-4,14 \\
(-3,73)\end{array}$ & $\begin{array}{c}1,19 \\
(25,32)\end{array}$ & $\begin{array}{c}0,60 \\
(3,54)\end{array}$ & -- & $\begin{array}{c}-0,07 \\
(-1,15)\end{array}$ & ,957 &, 953 & 279,8 & 0,64 & -- \\
\hline \multicolumn{11}{|l|}{ JEUNES } \\
\hline $\begin{array}{l}\text { H3 (concur- } \\
\text { rencés par } \\
\text { les femmes) }\end{array}$ & $\begin{array}{l}-38,84 \\
(-4,68)\end{array}$ & $\begin{array}{c}1,81 \\
(25,14)\end{array}$ & $\begin{array}{c}1,33 \\
(7,05)\end{array}$ & $\begin{array}{c}0,50 \\
(3,60)\end{array}$ & $\begin{array}{c}-1,72 \\
(-8,37)\end{array}$ & ,975 & 970 & 205,6 & 1,99 & ,46 \\
\hline $\begin{array}{l}\mathrm{H} 2 \text { (segmen- } \\
\text { tation) }\end{array}$ & $\begin{array}{l}-10,85 \\
(-8,32)\end{array}$ & $\begin{array}{c}1,72 \\
(21,07)\end{array}$ & $\begin{array}{c}0,03 \\
(0,06)\end{array}$ & $\begin{array}{c}0,44 \\
(3,72)\end{array}$ & $\begin{array}{l}-1,67 \\
(-8,22)\end{array}$ & ,976 &, 971 & 211,1 & 2,32 &, 92 \\
\hline $\begin{array}{l}\mathrm{H} 4 \text { (concur- } \\
\text { rence large) }\end{array}$ & $\begin{array}{l}-5,31 \\
(-0,34)\end{array}$ & $\begin{array}{c}1,86 \\
(14,78)\end{array}$ & $\begin{array}{c}1,57 \\
(3,07)\end{array}$ & $\begin{array}{c}-0,05 \\
(-0,26)\end{array}$ & $\begin{array}{c}-1,73 \\
(-6,55)\end{array}$ & ,956 &, 952 & 125.7 & 1,49 &,- 82 \\
\hline $\begin{array}{l}\text { HI (compen- } \\
\text { sation) }\end{array}$ & $\begin{array}{c}-9,36 \\
(-6,00)\end{array}$ & $\begin{array}{c}1,89 \\
(21,94)\end{array}$ & $\begin{array}{c}1,69 \\
(8,48)\end{array}$ & -- & $\begin{array}{l}-1,74 \\
(-6,83)\end{array}$ & ,960 & ,954 & 175,0 & 1,49 & -- \\
\hline
\end{tabular}

* En raison du problème de données non comparables pour la population active des jeunes, cette régression porte sur la sous-période 1976-1988.

17. Les sources de données sont la Gazette Officielle du Québec pour les données sur le salaire minimum, Emploi, gains et durée du travail (Statistique Canda, cat. no. 72-002) pour le salaire horaire manufacturier, et Statistique Canada, cat. no. 71-001, La Population active, pour les autres variables. 
exception, le nombre d'observations est de 42 pour les femmes et de 26 pour les jeunes. Les périodes considérées sont respectivement de 1968 à 1988 pour les femmes et de 1976 à 1988 pour les jeunes.

\section{Le choix de la variable d'offre}

Dans le cas des femmes, nous trouvons tout d'abord que l'hypothèse $\mathrm{H} 3$ est rejetée. En effet, si on consulte la première rangée du tableau 2, on trouve que le coefficient de la variable de population active relative est du bon signe mais qu'il est non significatif $(t=1,55)$. $\mathrm{H} 2$ par contre (deuxième rangée), est l'hypothèse qui affiche la meilleure performance sur le plan statistique. Les $\mathrm{R}^{2}$ sont plus élevés (,988 et, 987$)$ que partout ailleurs, le F est aussi plus élevé $(789,6)$. Le Durbin-Watson ayant une valeur de 1,93 indique l'absence d'autocorrélation des résidus. Dans le cas de $\mathrm{H} 1$ par ailleurs, (quatrième rangée), on dénote un sérieux problème d'autocorrélation des résidus (D.-W. $=0,64$ ). Finalement, si on compare la statistique t qui affecte le coefficient de la variable de population active relative $\left(P A R_{k}\right)$, on trouve qu'en $\mathrm{H} 2$ elle dépasse toutes les autres de par sa taille $(10,07)$, y inclus la spécification $\mathrm{H} 4$ qui lui offre le plus de concurrence $(8,86)$. En somme, le modèle d'estimation s'appuyant sur l'hypothèse $\mathrm{H} 2 \mathrm{~s}$ 'avère supérieur à toutes les autres spécifications. Dans les étapes qui suivent nous nous appuyerons sur cette spécification pour estimer l'incidence du salaire minimum sur le taux de chômage des femmes.

Dans le cas des jeunes, nous trouvons tout d'abord que les spécifications $\mathrm{H} 4 \mathrm{et}$ $\mathrm{H} 1$ sont à rejeter (deux dernières rangées du tableau 2). En effet les $\mathrm{R}^{2}$, les $\mathrm{F}$ et les Durbin-Watson y sont moins performants. Dans le cas de l'hypothèse $\mathrm{H} 4$ tout particulièrement, il arrive que le coefficient de la variable de population active est négatif et non significatif $(t=-0,26)$. À première vue, par contre, il semble que les spécifications $\mathrm{H} 3$ et $\mathrm{H} 2$ soient en forte concurrence l'une avec l'autre. D'une part, les $R^{2}$ et la statistique $F$ sont légèrement plus élevés en $H 2$ qu'en $H 3$. D'autre part, la statistique de Durbin-Watson tombe dans une région d'indétermination dans le cas de la spécification $\mathrm{H} 2$ alors qu'elle indique l'absence d'autocorrélation des résidus dans le cas de $\mathrm{H} 3$. Finalement, la colinéarité entre la variable de salaire minimum et la variable de population active est si élevée dans le cas de l'hypothèse H2 ( $r=.92)$ que le coefficient de la variable de salaire minimum perd toute sa précision $(t=0,06)^{18}$. Dans la mesure où il convient de rechercher une variance minimale pour les fins d'évaluation de l'incidence du salaire minimum sur le chômage, il ressort de cette analyse que la spécification $\mathrm{H} 3$ est préférable à la spécification H2. Dans les étapes qui suivent nous nous servirons donc de la spécification $\mathrm{H} 2$ pour les femmes et de la spécification $\mathrm{H} 3$ pour les jeunes ${ }^{19}$.

18. De 1976 à 1986 plus particulièrement, le salaire minimum relatif et l'importance relative des jeunes dans la population active totale au Québec connaissent, simultanément, un déclin continu et régulier.

19. Le fait que les femmes sentent moins les effets de la concurrence des jeunes peut s'expliquer en partie tout au moins par le fait qu'elles représentent une masse plus importante sur le marché du travail (40\% du marché) que les jeunes $(20 \%)$. 


\section{TABLEAU 3}

ÉTUDE DE CERTAINES HYPOTHÈSES RELATIVES À LA FORME DE LA RELATION

\begin{tabular}{|c|c|c|c|c|c|c|c|c|c|}
\hline & $a_{\mathrm{o}}$ & $T C T$ & $S M R$ & $P A R$ & $D S$ & $\mathbf{R}^{2}$ & $\overline{\mathbf{R}}^{2}$ & $\mathrm{~F}$ & D.-W. \\
\hline $\begin{array}{c}\text { FEMMES } \\
1968-88\end{array}$ & $\begin{array}{l}-15,36 \\
(-12,22)\end{array}$ & $\begin{array}{c}0,96 \\
(28,11)\end{array}$ & $\begin{array}{c}1,25 \\
(11,37)\end{array}$ & $\begin{array}{c}0,28 \\
(10,07)\end{array}$ & $\begin{array}{l}-0,10 \\
(-1,04)\end{array}$ & ,988 & ,987 & 789,6 & 1,93 \\
\hline $\begin{array}{c}\text { JEUNES } \\
1976-88\end{array}$ & $\begin{array}{l}-38,84 \\
(-4,68)\end{array}$ & $\begin{array}{c}1,81 \\
(25,14)\end{array}$ & $\begin{array}{c}1,33 \\
(7,05)\end{array}$ & $\begin{array}{c}0,50 \\
(3,60)\end{array}$ & $\begin{array}{c}-1,72 \\
(-8,37)\end{array}$ & 975 &, 970 & 205,6 & 1,99 \\
\hline & $a_{0}$ & TCT & $S M R^{2}$ & $P A R$ & $D S$ & $\mathbf{R}^{2}$ & $\overline{\mathbf{R}}^{2}$ & $F$ & D.-W. \\
\hline $\begin{array}{c}\text { FEMMES } \\
1968-88\end{array}$ & $\begin{array}{l}-12,23 \\
(-12,05)\end{array}$ & $\begin{array}{c}0,96 \\
(28,55)\end{array}$ & $\begin{array}{l}, 136 \\
(11,51)\end{array}$ & $\begin{array}{c}0,27 \\
(9,98)\end{array}$ & $\begin{array}{c}-0,10 \\
(-1,08)\end{array}$ & ,989 & ,987 & 804,5 & 1,99 \\
\hline $\begin{array}{c}\text { JEUNES } \\
1976-88\end{array}$ & $\begin{array}{l}-38,79 \\
(-4,71)\end{array}$ & $\begin{array}{c}1,82 \\
(25,31)\end{array}$ & $\begin{array}{c}, 147 \\
(7,13)\end{array}$ & $\begin{array}{c}0,54 \\
(4,04)\end{array}$ & $\begin{array}{c}-1,71 \\
(-8,40)\end{array}$ & ,976 & ,976 & 208,9 & 1,99 \\
\hline
\end{tabular}

TABLEAU 4

TEST DE L'HYPOTHÈSE DE LA SYMÉTRIE DES EFFETS DU SALAIRE MINIMUM FEMMES, QUÉBEC, 1968-1976

\begin{tabular}{c|ccccccccccc}
\hline \hline \multicolumn{2}{c}{$a_{\mathrm{o}}$} & $T C T$ & $S M R^{2}$ & $P A R$ & $D S$ & $D 76$ & $\mathrm{R}^{2}$ & $\mathrm{R}^{2}$ & $\mathrm{~F}$ & D.-W. \\
\hline $\begin{array}{c}\text { Modèle } \\
\text { linéaire } \\
(S M R)\end{array}$ & $-15,49$ & 0,96 & 1,26 & 0,28 & $-0,10$ & $-0,004$ &, 988 &, 987 & 614,8 & 1,93 \\
$\begin{array}{c}\text { Modèle } \\
\text { quadratique } \\
\left(S M R^{2}\right)\end{array}$ & $(-8,29)$ & $(25,36)$ & $(8,63)$ & $(7,56)$ & $(-1,03)$ & $(-0,10)$ & & & & \\
\hline
\end{tabular}




\section{La forme fonctionnelle}

Suite aux diverses estimations qui ont été effectuées pour identifier la forme la plus appropriée de relation entre le salaire minimum et le chômage, il est ressorti de ces travaux que la forme quadratique simple $\left(S M R^{2}\right)$ était, à toutes fins pratiques, la plus efficace. Le tableau 3 rapporte à cet effet les résultats comparatifs des estimations avec les meilleurs résultats obtenus précédemment.

Les deux premières lignes du tableau 3 rappellent les meilleurs résultats issus du tableau 2. Les deux dernières lignes présentent les résultats d'estimation lorsque la variable de salaire minimum est au carré. L'hypothèse de non linéarité de la relation entre le salaire minimum et le taux de chômage est confirmée. Par comparaison, ces résultats s'avèrent également légèrement supérieurs ou meilleurs. Les $R^{2}$ sont supérieurs ou égaux aux précédents. Les $F$ sont légèrement meilleurs, de même que les statistiques Durbin-Watson (désormais toutes deux égales à 1,99). Finalement, les statistiques $t$ qui affectent les coefficients de la variable de salaire minimum relatif sont plus élevées dans sa formulation non linéaire que dans sa formulation linéaire. En somme, il apparaît que la forme quadratique génère des estimations plus précises de l'incidence du salaire minimum sur le chômage des jeunes et des femmes au Québec.

\section{La symétrie des effets}

Afin de vérifier si les baisses et les hausses du salaire minimum avaient les mêmes effets mais en sens inverse, nous avons incorporé au modèle initial de détermination du taux de chômage des femmes, une variable dichotomique croisée avec la variable de salaire minimum à partir du premier semestre de l'année 1976 jusqu'à la fin de 1988. S'il arrivait que le coefficient de cette variable (D76) soitélevé et significatif sur le plan statistique, cela signifierait que les effets du salaire minimum sur le chômage seraient asymétriques et que les résultats seraient variables selon qu'on procède à des estimations en période où le salaire minimum relatif est en hausse (1968-1975) ou en période où celui-ci est en baisse (1976$1988)^{20}$. Dans l'éventualité où le coefficient de cette variable serait petit et non significatif, cela signifierait au contraire que les effets sont parfaitement symétriques et indépendants du type d'observation. Le tableau 4 rapporte ces résultats d'estimation pour le modèle linéaire comme pour le modèle non linéaire.

Dans un cas comme dans l'autre, la variable croisée prend une valeur très faible, négative dans un cas, positive dans l'autre et non significative dans les deux cas. Les t sont respectivement de $-0,10$ et de 0,06 . Les autres coefficients estimés sont par ailleurs très peu affectés par cette transformation dont les coefficients des variables de salaire minimum relatif. Nous en concluons, dans le cas des femmes plus

20. De 1968 à 1975 , le salaire minimum relatif est passé de 0,46 à 0,55 . De 1976 à 1988 , il est passé de 0,55 a 0,37 . La période de baisse du salaire minimum relatif s'est toutefois concentrée entre 1976 et 1989. En 1987 et 1988, on a assisté à des hausses et des baisses du salaire minimum relatif par semestre. 
particulièrement, à la symétrie des effets à la baisse comme à la hausse du salaire minimum sur leur taux de chômage ${ }^{21}$.

\section{LA STABILITÉ DES RÉSULTATS}

Tel qu'indiqué en introduction, la question de savoir si les résultats sont stables dans le temps nous intéressait grandement. Nous avons donc fait comme si l'étude avait été publiée à différentes années au cours des années 80 . C'est ainsi que nous avons répété l'exercice neuf fois soit sur la période 1968-1981, 1968-1982, 19681983 et ainsi de suite jusqu'à la période 1968-1988 dans le cas des femmes.Dans le cas des jeunes, nous avons appliqué la même méthode consistant à intégrer successivement les neuf dernières observations (1984II-1988II) plutôt que les neuf dernières années. Le tableau 5 présente les coefficients de régression de la variable de salaire minimum et leurs t correspondants pour les deux groupes de maind'oeuvre à l'étude et les deux formes considérées.

Les estimations et les conclusions des études auraient très peu changé à travers le temps. C'est ainsi par exemple que si on suppose un délai de publication de deux ans par rapport aux dernières données disponibles, les conclusions d'une étude publiée en 1982 auraient été, dans sa version linéaire, qu'un accroissement de 10 points de pourcentage dans le salaire minimum relatif aurait pour effet d'accroître le chômage des jeunes et des femmes de 1,2 point de pourcentage dans chacun des cas (voir résultats pour la période 1968-1980). Ces conclusions seraient de 1,3 point de pourcentage pour les femmes et de 1,1 point de pourcentage pour les jeunes si l'étude avait été réalisée et publiée en 1985 (période 1968-1983). Dans sa version linéaire la plus récente, ces conclusions sont de 1,3 point de pourcentage pour chacun de ces groupes. À la différence des résultats plus anciens cependant, on note que les statistiques t sont plus élevées pour les résultats les plus récents; que l'on prenne la forme linéaire ou la forme non linéaire pour référence. Ceux-ci auraient donc tendance à être de plus en plus précis au fur et à mesure que nous avançons dans le temps.

Dès lors, si on retient l'estimation la plus récente pour la forme la plus performante $\left(S M R^{2}\right)$, nous avons un coefficient de 0,136 pour les femmes et de 0,147 pour les jeunes. Ces coefficients s'interprètent de la façon suivante: la variation de chômage associée à une variation de 10 points dans le salaire minimum relatif est égale à deux fois le coefficient multiplié par la valeur du salaire minimum relatif, multiplié par dix ${ }^{22}$. C'est ainsi par exemple qu'à un taux de salaire minimum relatif de $50 \%$, l'impact estimé est de 10 fois le coefficient $(2 * 0,136 * 0,50 * 10=1,36$ dans le cas des femmes et $2 * 0,147 * 0,50 * 10=1,47$ dans le cas des jeunes). Le tableau 6

21. Dans le cas des jeunes, nous ne pouvons tester la symétrie des effets, mais les résultats obtenus sur une période de baisse du salaire minimum relatif nous font conclure que ces baisses ont pu contribuer à réduire leur taux de chômage.

22. Rappelons que pour faciliter l'interprétation des résultats, le quotient du salaire minimum sur le salaire horaire dans le secteur manufacturier avait été multiplié par 10, d'où le fait de n'avoir qu'à multiplier par 10 plutôt que par 100. Par ailleurs, la dérivée de $\mathrm{X}$ au carté étant égale à $2 \mathrm{X}$, il convient de multiplier le coefficient de la variable de salaire minimum au carré par deux. 


\section{TABLEAU 5}

COEFFICENTS ET STATISTIQUES $t$ DE LA VARIABLE DE SALAIRE MINIMUM, DIFFÉRENTES PÉRIODES D'OBSERVATION

\begin{tabular}{|c|c|c|c|c|c|}
\hline \multicolumn{3}{|c|}{ FEMMES } & \multicolumn{3}{|c|}{ JEUNES } \\
\hline Période & $\begin{array}{c}S M R \\
\text { (linéaire) }\end{array}$ & $\begin{array}{c}S M R^{2} \\
\text { (quadratique) }\end{array}$ & Période & $\begin{array}{c}S M R \\
\text { (linéaire) }\end{array}$ & $\begin{array}{c}S M R^{2} \\
\text { (quadratique) }\end{array}$ \\
\hline $1968-1980$ & $\begin{array}{c}1,21 \\
(4,89)\end{array}$ & $\begin{array}{l}0,118 \\
(4,82)\end{array}$ & $1976 \mathrm{I}-1984 \mathrm{II}$ & $\begin{array}{c}1,19 \\
(3,50)\end{array}$ & $\begin{array}{l}0,129 \\
(3,35)\end{array}$ \\
\hline $1968-1981$ & $\begin{array}{c}1,21 \\
(5,91)\end{array}$ & $\begin{array}{l}0,119 \\
(5,84)\end{array}$ & 1976I-1985I & $\begin{array}{c}1,12 \\
(4,11)\end{array}$ & $\begin{array}{l}0,122 \\
(3,96)\end{array}$ \\
\hline $1968-1982$ & $\begin{array}{c}1,35 \\
(7,82)\end{array}$ & $\begin{array}{l}0,134 \\
(7,61)\end{array}$ & $1976 \mathrm{I}-1985 \mathrm{II}$ & $\begin{array}{c}1,28 \\
(5,30)\end{array}$ & $\begin{array}{l}0,141 \\
(5,12)\end{array}$ \\
\hline $1968-1983$ & $\begin{array}{c}1,34 \\
(8,98)\end{array}$ & $\begin{array}{l}0,137 \\
(8,73)\end{array}$ & $1976 \mathrm{I}-1986 \mathrm{I}$ & $\begin{array}{c}1,12 \\
(5,06)\end{array}$ & $\begin{array}{l}0,125 \\
(4,96)\end{array}$ \\
\hline $1968-1984$ & $\begin{array}{c}1,20 \\
(9,10)\end{array}$ & $\begin{array}{l}0,127 \\
(9,18)\end{array}$ & $1976 \mathrm{I}-1986 \mathrm{II}$ & $\begin{array}{c}1,21 \\
(5,85)\end{array}$ & $\begin{array}{l}0,135 \\
(5,78)\end{array}$ \\
\hline $1968-1985$ & $\begin{array}{c}1,21 \\
(10,84)\end{array}$ & $\begin{array}{c}0,131 \\
(10,84)\end{array}$ & $1976 \mathrm{I}-1987 \mathrm{I}$ & $\begin{array}{c}1,28 \\
(6,11)\end{array}$ & $\begin{array}{l}0,143 \\
(6,15)\end{array}$ \\
\hline $1968-1986$ & $\begin{array}{c}1,21 \\
(11,88)\end{array}$ & $\begin{array}{c}0,132 \\
(11,87)\end{array}$ & 1976I-1987II & $\begin{array}{c}1,28 \\
(6,45)\end{array}$ & $\begin{array}{l}0,143 \\
(6,51)\end{array}$ \\
\hline $1968-1987$ & $\begin{array}{c}1,25 \\
(11,23)\end{array}$ & $\begin{array}{c}0,136 \\
(11,33)\end{array}$ & $1976 \mathrm{I}-1988 \mathrm{I}$ & $\begin{array}{c}1,36 \\
(6,98)\end{array}$ & $\begin{array}{l}0,150 \\
(7,09)\end{array}$ \\
\hline $1968-1988$ & $\begin{array}{c}1,25 \\
(11,37)\end{array}$ & $\begin{array}{c}0,136 \\
(11,51)\end{array}$ & 1976I-1988II & $\begin{array}{c}1,33 \\
(7,05)\end{array}$ & $\begin{array}{l}0,147 \\
(7,13)\end{array}$ \\
\hline
\end{tabular}


TABLEAU 6

INCIDENCE D'UNE VARIATION DE 10 POINTS DE POURCENTAGE DANS LE SALAIRE MINIMUM RELATIF SUR LE TAUX DE CHÔMAGE DES JEUNES ET DES FEMMES

\begin{tabular}{|c|c|c|c|c|c|c|c|c|c|c|c|c|}
\hline \multirow[b]{2}{*}{ FEMMES } & \multicolumn{2}{|c|}{$\begin{array}{c}S M R \\
\text { incidence } \\
\end{array}$} & \multicolumn{2}{|c|}{$\begin{array}{c}S M R \\
\text { incidence } \\
\end{array}$} & \multicolumn{2}{|c|}{$\begin{array}{c}S M R \\
\text { incidence } \\
\end{array}$} & \multicolumn{2}{|c|}{$\begin{array}{c}S M R \\
\text { incidence }\end{array}$} & \multicolumn{2}{|c|}{$\begin{array}{c}S M R \\
\text { incidence }\end{array}$} & \multicolumn{2}{|c|}{$\begin{array}{c}S M R \\
\text { incidence } \\
\end{array}$} \\
\hline &, 30 &, 81 &, 35 & ,95 &, 40 & 1,09 &, 45 & 1,22 &, 50 & 1,36 &, 55 & 1,50 \\
\hline &, 31 &, 84 &, 36 & ,98 & ,41 & 1,12 & ,46 & 1,25 &, 51 & 1,39 & ,56 & 1,52 \\
\hline &, 32 &, 87 & 37 & 1,01 & 42 & 1,14 & 47 & 1,28 &, 52 & 1,41 &, 57 & 1,55 \\
\hline &, 33 &, 89 &, 38 & 1,03 & ,43 & 1,17 & ,48 & 1,31 &, 53 & 1,44 &, 58 & 1,58 \\
\hline &, 34 &, 92 &, 39 & 1,06 &, 44 & 1,20 &, 49 & 1,33 &, 54 & 1,47 & .59 & 1,60 \\
\hline \multirow[t]{5}{*}{ JEUNES } &, 30 &, 88 &, 35 & 1,03 &, 40 & 1,18 &, 45 & 1,32 &, 50 & 1,47 &, 55 & 1,62 \\
\hline & ,31 &, 91 &, 36 & 1,06 & ,41 & 1,21 & ,46 & 1,35 & .51 & 1,50 & .56 & 1,65 \\
\hline &, 32 &, 94 & 37 & 1,09 & ,42 & 1,23 &, 47 & 1,38 & .52 & 1,53 & .57 & 1,68 \\
\hline &, 33 & ,97 &, 38 & 1,12 & ,43 & 1,26 & ,48 & 1,41 &, 53 & 1,56 &, 58 & 1,71 \\
\hline & .34 & 1,00 &, 39 & 1,15 &, 44 & 1,29 &, 49 & 1,44 &, 54 & 1,59 &, 59 & 1,73 \\
\hline
\end{tabular}


reproduit les variations de chômage associées à une variation de 10 points dans le salaire minmum relatif pour des taux de salaire minimum relatifs initiaux variant entre $30 \%$ et $59 \%$ du salaire horaire manufacturier au Québec ${ }^{23}$.

Ce tableau indique une forte sensibilité des coefficients d'ajustement du chômage au salaire minimum. Dans le cas des femmes par exemple, ils peuvent varier de 0,81 lorsque le salaire minimum est à $30 \%$ du salaire manufacturier à 1,60 lorsque celui-ci représente $59 \%$ du salaire manufacturier. Les chiffres correspondants pour les jeunes sont de 0,88 et 1,73 respectivement. Cette variabilité est cependant quelque peu théorique car les variations effectives du chômage dépendent non pas des coefficients d'ajustement marginaux (ceux du tableau 6) mais des coefficients moyens. Par exemple, si nous trouvons un coefficient de 1,25 dans le cas de l'estimation linéaire pour les femmes c'est en grande partie attribuable au fait que la valeur moyenne du salaire minimum relatif sur l'ensemble de la période est de 0,46 (voir tableau 6). Cette valeur moyenne a très peu tendance à changer à travers le temps compte tenu du poids des valeurs passées du salaire minimum.

En somme, bien que l'hypothèse ait été soutenue à l'effet qu'une hausse de salaire minimum qui s'applique à des taux déjà élevés a plus d'impact et qu'une baisse du salaire minimum qui s'applique à des taux déjà faibles a moins d'impact, il reste que l'approximation linéaire apparaît tout à fait justifiée en temps normal. Nos meilleures estimations à cet égard sont un accroissement de 1,25 point de pourcentage dans le taux de chômage des femmes et de 1,33 point de pourcentage dans le taux de chômage des jeunes pour une variation de 10 points de pourcentage dans le taux de salaire minimum relatif ${ }^{24}$. La réduction du chômage des jeunes et des femmes au Québec suite à la réduction du salaire minimum relatif au cours de la période 1976-1988 peut donc être évaluée à 2,4 points de pourcentage pour les jeunes et 2,3 points de pourcentage pour les femmes ${ }^{25}$.

Bien que ce type de mesure est courant dans la littérature économique, en pratique il apparaît que les décisions ne sont pas prises comme telles sur le taux de salaire minimum relatif. Nous avons donc imaginé trois scénarios de décisions ou politiques de salaire minimum qui permettent de se représenter plus clairement leurs effets sur le chômage des jeunes et des femmes au Québec. Nous supposons tout d'abord qu'il s'agit d'une politique constante sur cinq ans et que le salaire horaire manufacturier augmente de $4 \%$ par année. Dans le premier scénario, le gouvernement déciderait d'appliquer une politique de salaire minimum qui consisterait à décréter des augmentations du salaire minimum systématiquement supérieures au

23. Dans les faits, il a varié entre $35 \%$ et $55 \%$.

24. Cousineau (1979) en arrivait à des estimations sensiblement plus élevées: 1,7 point de pourcentage pour les jeunes, 2,3 points de pourcentage pour les femmes.

25. Les estimations de Myatt et Murrell (1990) quant à la réduction de l'écart entre le taux de chômage des femmes et des hommes au Canada est de 3 points de pourcentage. Les estimations de Coe (1990) quant à la réduction du taux de chômage total pour l'ensemble du Canada sont de 1 point de pourcentage. 
TABLEAU 7

SCÉNARIOS DE POLITIQUES DE SALAIRE MINIMUM ET INCIDENCE SUR LE CHÔMAGE DES JEUNES ET DES FEMMES AU QUÉBEC

\begin{tabular}{|c|c|c|c|c|c|c|}
\hline \multicolumn{6}{|c|}{ DIFFÉRENTIELLE POSITIVE } & \multirow{2}{*}{$\begin{array}{c}\text { Indexation } \\
\text { partielle }\end{array}$} \\
\hline & $1 \%$ & $2 \%$ & $3 \%$ & $4 \%$ & Gel & \\
\hline \multicolumn{7}{|l|}{$\begin{array}{l}\text { Variation } \\
\text { du chômage }\end{array}$} \\
\hline JEUNES & 0,3 & 0,5 & 0,8 & 1,1 & $-0,9$ & $-0,5$ \\
\hline FEMMES & 0,3 & 0,5 & 0,8 & 1,0 & $-0,9$ & $-0,5$ \\
\hline
\end{tabular}

salaire horaire dans le secteur manufacturier $(1 \%, 2 \%, 3 \%$ ou $4 \%$ de plus, soit des augmentations annuelles moyennes de $5 \%, 6 \%, 7 \%$ ou $8 \%$ respectivement). Dans le deuxième scénario, nous envisageons le cas d'un gel du salaire minimum pendant une période de cinq ans. Dans le dernier scénario, nous envisageons une situation d'indexation partielle, soit des augmentations du salaire minimum de $2 \%$ par année alors que le salaire horaire moyen dans le secteur manufacturier augmenterait de $4 \%$ par année ${ }^{26}$. Les résultats de ces scénarios sont présentés au tableau 7. On y observe essentiellement qu'un scénario d'accroissements supérieurs du salaire minimum par rapport au salaire manufacturier aurait pour conséquence d'accroître le chômage des jeunes et des femmes entre 0,3 point et 1,1 point de pourcentage selon la différentielle adoptée.

Dans le cas du gel du salaire minimum pendant une période de cinq ans et à supposer qu'entretemps le salaire manufacturier augmente de $4 \%$ par année, l'effet sur le chômage des jeunes et des femmes au Québec est estimé à une réduction de 0,9 point de pourcentage dans chacun des cas. Finalement, dans le scénario intermédiaire d'indexation partielle, l'effet estimé en est un d'une réduction d'un demi-point de pourcentage sur l'ensemble de la période pour chacun des deux groupes visés.

\section{CONCLUSION}

Le salaire minimum renferme de bons et de mauvais côtés. D'une part, il occasionne des coûts dont la détermination de niveaux d'emplois inférieurs et de taux de chômage supérieurs à ce qu'ils seraient en l'absence de salaire minimum.

26. Ce scénario pourrait correspondre à une situation où l'inflation augmente de $2 \%$. Dans ces circonstances, le salaire minimum serait indexé au coût de la vie. 
D'autre part, le salaire minimum, avec les autres conditions minimales de travail, offre des garanties contre l'exploitation de la main-d'oeuvre, de même qu'il assure à une partie de cette main-d'oeuvre des revenus minimaux (en l'absence d'une formule de revenu minimum garanti généralisée). Selon Lang (1987), il peut même avoir certaines vertus au niveau de l'efficacité économique. Les politiciens doivent donc en soupeser les coûts et les bénéfices ${ }^{27}$. Cette étude de l'incidence du salaire minimum sur le chômage des jeunes et des femmes au Québec porte donc essentiellement sur ses coûts.

Sur le plan général tout d'abord nous avons passé en revue quelques-unes des critiques soulevées à l'égard des études d'impact du salaire minimum sur le chômage. Nos principales conclusions ont été à l'effet que les études économétriques de l'incidence du salaire minimum sur le chômage fournissent une évaluation minimale des effets du salaire minimum sur l'emploi et le degré de sous-emploi dans une économie, une fois tous les ajustements du marché complétés.

Par la suite nous avons considéré quelques améliorations possibles à apporter aux modèles existant pour trouver 1) qu'il fallait chercher à mieux tenir compte des développements au niveau du chômage structurel ou «naturel»; 2) que la variable d'offre de travail pouvait être mais n'était pas nécessairement une variable de population active relative du seul groupe à l'étude; 3) qu'on était en droit de s'attendre à ce que la relation entre le salaire minimum et le chômage soit non linéaire.

Sur le plan empirique nous avons commencé par chercher la variable d'offre de travail la plus pertinente puis nous avons successivement vérifié l'hypothèse de non linéarité, la symétrie des effets et la stabilité des estimations. Les meilleurs résultats d'estimation sont apparus comme étant ceux de l'application des moindres carrés ordinaires sur des données semi-annuelles pour une forme non linéaire (quadratique) du salaire minimum. L'étude de sensibilité des estimations nous a montré toutefois qu'elles étaient relativement insensibles à la période d'estimation et que la forme linéaire pouvait jouer le rôle de bonne approximation des effets du salaire minimum sur le chômage des jeunes et des femmes au Québec. Ces estimations sont à l'effet qu'un accroissement de 10 points de pourcentage dans le salaire minimum relatif a pour effet d'accroître le taux de chômage des femmes et des jeunes de l'ordre de 1,25 et 1,33 point de pourcentage respectivement. Dans le cas des femmes, nous avons pu vérifier que cet effet était, à toutes fins pratiques, parfaitement symétrique. Dans le cas des jeunes, il est aussi apparu que leur taux de chômage était sensible aux baisses du salaire minimum relatif. En fait, les baisses observées dans le salaire

27. Et il semble bien qu' ils le fassent jusqu'à un certain point. Blais et al. (1989) en concluent d'ailleurs qu'ils tiennent compte de la conjoncture économique, des groupes de pression, de leur idéologie et de l'information scientifique autour de cette question. Pour notre part, nous avons essayé de voir si les variations annuelles du salaire minimum étaient déterminées comme si elles suivaient les lois du marché (courbe de Phillips). Nos résultats d'estimation corroborent cette hypothèse et montrent que les ajustements du salaire minimum sont plus rapides et plus sensibles aux variables de marché que dans le cas des variations du salaire horaire manufacturier. 
minimum relatif au cours des années 1976-1988 ont pu contribuer à cet égard à réduire le chômage des femmes et des jeunes au Québec de l'ordre de 2,3 et 2,4 points de pourcentage respectivement. Le tableau de la demière section explicite quant à lui, l'effet de certains scénarios de politiques de salaire minimum sur le chômage. On y découvre notamment qu'une politique qui viserait à accorder pendant cinq années consécutives des augmentations de salaire minimum supérieures à celles du salaire manufacturier accroîtrait de 0,3 à 1 point de pourcentage ou un peu plus le chômage des jeunes et des femmes au Québec.

\section{BIBLIOGRAPHIE}

Benhayoun, Gilbert, (1990), «Salaire minimum et emploi des jeunes», Notes de recherche, Centre d'économie régionale, Faculté d' Économie Appliquée, Aixen-Provence, janvier 1990, 57 pages.

Blais, André, Jean-Michel Cousineau et Kenneth McRoberts, (1989), «The Determinants of Minimum Wage Rates» Public Choice, 62, pp. 15 à 24.

Berndt, Ernst R. et Laurence R. Christansen, (1973), «The Translog Function and The Substitution of Equipment, Structures, and Labor in U.S. Manufacturing, 1929-68», Journal of Econometrics 1, 1973, pp. 81 à 114.

Boutin, JEAN-GuY, (1974), «Une étude des caractéristiques socio-économiques des travailleurs rémunérés autour du salaire minimum», Ministère des Affaires Sociales, Gouvernement du Québec.

Brechling, Frank D.B., (1965), «The Relationship Between Output and Employment in British Manufacturing Industries», The Review of Economics and Statistics, juillet, pp. 187 à 216.

Brown, Charles, Curtis Gilroy et Andrew Cohen, (1982), «The Effect of the Minimum Wage on Employment and Unemployment», Journal of Economic Literature, vol. XX, no. 2, Juin 1982, pp. 487 à 528.

Clark, Kim B. et Richard B. Freeman, (1980), «How Elastic is the Demand for Labor?», The Review of Economics and Statistics, novembre 1980,pp. 509 à 520.

CoE, David T., (1990), «Structural Determinants of the Natural Rate of Unemployment in Canada», International Monetary Fund Staff Papers, vol. 37, no. 1, mars 1990, pp. 94 à 115.

COURNOYER, MiCHEL, (1988), «Les caractéristiques principales des personnes à bas salaires au Québec», Interventions économiques, no. 19.

COUSINEAU, JEAN-MICHEL, (1979), «Impact du salaire minimum sur le chômage des jeunes et des femmes au Québec», Relations Industrielles, vol. 34, no.3, pp. 403 à 416 .

Fortin, Pierre, (1989), «How 'Natural' is Canada High Unemployment Rate», European Economic Review, vol. 33, no. 1, janvier 1989, pp. 89 à 110. 
Grenier, Gilles et Marc Seguin, (1990), «L'incidence du salaire minimum sur le marché du travail des adolescents au Canada: une reconsidération des résultats empiriques», texte de ce symposium.

Gunderson, Morley et Craigg W. Riddell, (1988), Labour Market Economics, 2ème édition, McGraw-Hill Ryerson, Montréal, pp. 171 à 176.

HAMERMESh, DANIEL S., (1988), «The Demand for Labor in the Long Run», dans Handbook of Labor Economics, ORLey C. Ashenfelter et Richard Layard (éds), vol. 1, ch. 8, North Holland, New York, pp. 429 à 471.

LANG, K., (1987), «Pareto Improving Minimum Wage Laws», Economic Inquiry, vol. XXV, janvier 1987.

MerCIER, JACQUeS, (1985), «Les effets du salaire minimum sur l'emploi des jeunes au Québec» Relations Industrielles, vol. 40, no.3, pp. 431 à 457.

Myatt, Anthony et David Murrell, (1990), «The Female/Male Unemployment Differential», Revue canadienne d'Economique, vol. XXIII, no. 2, mai 1990, pp. 312 à 322.

NADIRI, M.I., (1968), «The Effects of Relative Prices and Capacity on the Demand for Labor in the U.S. Manufacturing Sector», The Review of Economic Studies, juillet 1968, pp. 273 à 288.

Oi, Walter, (1962), «Labor as a Quasi-Fixed Factor», The Journal of Political Economy, vol. 70, no. 6, décembre 1962, pp. 533 à 555.

WEST, E.G. et M. McKeE.(1980), Le salaire minimum: nouveauxaspects théoriques et applications, Conseil économique du Canada et Institut de recherche sur la politique économique, Ottawa. 